\title{
Un análisis de la producción y el desempleo en Nicaragua 1980-2014
}

\author{
An analysis of production and unemployment in Nicaragua 1980-2014
}

Oslund Rains Franklin Sam ${ }^{1}$

\section{Resumen}

En este artículo, se han expuesto las tres especificaciones empíricas desarrollados por Arthur Okun (Okun, 1962), economista norteamericano, quien estableció la relación entre crecimiento productivo y desempleo de Estados Unidos a partir de los datos recogidos durante los años 50. La metodología es cuantitativa al partir de la ecuación primeras diferencias, para estimar la relación empírica entre producción y empleo, utilizando datos desde la década de los 80 hasta el 2014 con frecuencia anual publicados por el Banco Central de Nicaragua, pero teniendo en cuenta que hubo cambios en el cálculo del año base de los datos del PIB real. Los cálculos efectuados en el desarrollo del modelo se hicieron utilizando el software libre econométrico Gretl disponible en internet. El enfoque estadístico se ha centrado en la introducción de una tendencia estocástica en el modelo de regresión, valorando los cambios estructurales de los últimos años en la economía nicaragüense. Se demostró que hay una relación positiva entre producción y desempleo, es decir, un aumento de un $1 \%$ del producto va acompañado con un aumento en el desempleo de un $0.18 \%$. Las variables se mueven en la misma dirección, contradiciendo la teoría económica.

Palabras clave: Producción; empleo; desempleo; crecimiento económico; Okun.

\section{Abstract}

This article, shows the three empirical specifications developed by Arthur Okun (Okun, 1962), an American economist, who established the relationship between productive growth and unemployment in the United States, based on the data collected during the 1950s. The methodology is quantitative because it used the equation first differences, to estimate the empirical relationship between production and employment, using data from 1980 to 2014 with annual frequency published by the Central Bank of Nicaragua, but taking into account that there were changes in the calculation of the base year of the real GDP data. The econometric software Gretl available on the internet was used to develop the model. The statistical approach has focused on the introduction of a stochastic trend in the regression model, assessing the structural changes of recent years in the Nicaraguan economy. It shows that there is a positive

1 Doctor en Ciencias Económicas, Director de adquisiciones de la Universidad de las Regiones Autónomas de la Costa Caribe Nicaragüense, correo: oslund.franklin@gmail.com, No. de Orcid: https://orcid.org/oooo-ooo2-7169-1967

Recibido: 05/02/2018 Aprobado: 30/04/2018

Franklin-Sam, 0. (2018). Un análisis de la producción y el desempleo en Nicaragua 1980-2014. Ciencia E Interculturalidad, 22(1), 137-145. D0l: 


\section{CIENCIAS SOCIALES}

relationship between production and unemployment, which implies that an increase of $1 \%$ of the product is accompanied by an increase in unemployment of $0.18 \%$. The variables move in the same direction, contradicting economic theory.

Key words: Production; employment; unemployment; economic growth; Okun

\section{Introducción}

La medición de la relación entre el crecimiento del producto de un país y el desempleo se considera uno de los temas tradicionales dentro de la macroeconomía. En el documento expuesto por Okun (1962), se brinda el soporte para efectuar tal medición. Esta relación postula la existencia de un movimiento inverso entre los cambios en la tasa de desempleo y los cambios en el producto real. Según Tobin (1980), constituye una de las regularidades empíricas más confiables dentro de la macroeconomía.

La relación existente entre la tasa de crecimiento y la tasa de desempleo se denomina "Ley de Okun", en honor al economista Arthur Okun en su artículo publicado en 1962 titulado:Potencial GNP: Its measuremente and significance, donde demuestra, mediante tres especificaciones econométricas dicha relación. En la mayoría de países, existe una relación entre la tasa de crecimiento del PIB y la variación de la tasa de desempleo. Okun, descubre esta relación en la década de los 6o's, donde sostiene básicamente que, un elevado crecimiento crea un incremento en el empleo del país y por ende, una disminución en la tasa de desempleo.

Pero ¿realmente estas variables tienen el mismo comportamiento que descubrió Okun para el caso de Nicaragua? En el presente artículo se demuestra que no necesariamente un aumento en la producción implica una disminución del desempleo. La tasa de desempleo es un objetivo básico de todo gobierno, por ello, es necesario conocer la magnitud en la que una economía debe crecer si desea mantener o reducir la tasa de desempleo en un país.

La consecución de un crecimiento económico dinámico, estable y sostenido en el tiempo, así como el mantenimiento de niveles de empleo aceptables son objetivos fundamentales de toda política económica de un país y a su vez requisitos indispensables para lograr una mejora en el bienestar socioeconómico de la población, con el propósito de disminuir la pobreza y fomentar el desarrollo armónico de la economía.

Se aplica la primera especificación planteada por Okun (primeras diferencias), para el caso de Nicaragua, utilizando datos anuales publicados por el Banco Central de Nicaragua desde la década de los 80’s hasta el 2014 sobre variables de producción y desempleo. 


\section{Revisión de literatura}

Arthur Okun (1962) en su el artículo titulado "Potential GNP: its measurement and significance", desarrolla tres especificaciones para medir la relación entre crecimiento y desempleo:

\section{Primeras diferencias}

$$
Y=\beta_{0}-\beta_{1} X
$$

La ecuación presenta la variación en la tasa de desempleo $(Y)$ como promedio de las variaciones porcentuales del producto real $(X)$, siendo $\beta 1$ el parámetro que mide la magnitud del cambio en $(Y)$, y $\beta o$ el intercepto. Dado un nivel de tecnología, los cambios trimestrales en la tasa de desocupación en puntos porcentuales, están relacionados con cambios trimestrales en porcentajes del producto real. Los resultados fueron: $Y$ $=0.3-0.3 X ;$ con un $r=0.79$ para la economía norteamericana.

Okun concluyó que cada 1\% de crecimiento en el producto de los Estados Unidos, el desempleo disminuiría en un tercio de punto porcentual y, por el contrario, un punto de aumento en la tasa de desempleo está asociado con una pérdida de $3.3 \%$ en el producto, de acuerdo al inverso del coeficiente $\beta 1$ estimado. 1/o.3 = 3.33.

\section{Relación de brechas}

Cambios a la tasa de desempleo (U) que dependen de la relación porcentual entre Producto Potencial y el Producto Efectivo u observado lo que se identifica como el GAP o brecha del producto:

$$
U=\alpha+\beta(G A P) ; \quad G A P=\frac{P I B_{P}-P I B_{r}}{P I B_{P}}
$$

Donde:

$\mathrm{U}=$ Tasa de desempleo.

$\mathrm{GAP}=$ Brecha del producto entre producto potencial y el producto efectivo u observado.

$\mathrm{PIB}_{P}=$ Producto Interno Bruto Potencial.

$\mathrm{PIB}_{r}=$ Producto Interno Bruto Efectivo u observado.

$\alpha=$ Constante. Donde puede ser tomado como proxy de la tasa natural de desempleo cuando GAP = o; ó PIBP = PIBr 


\section{CIENCIAS SOCIALES}

$\beta=$ Es el parámetro que mide el impacto de los cambios en la brecha sobre los cambios en U.

El resultado bajo este método fue: $\mathrm{U}=3.72+0.36(\mathrm{GAP})$.

Ahora $\alpha=3.72$ representa bajo el caso de $\beta=0$, un proxy de la tasa natural de desempleo de Estados Unidos.

El valor que acompaña el GAP del producto (o.36) indica la disminución (aumento) que experimentara la tasa de desempleo por debajo (arriba) de la tasa natural de desempleo, cuando la brecha del producto es negativa (positivo), lo cual es indicativo a su vez de que el producto efectivo tuvo una tasa de crecimiento mayor (menor) a la tasa de crecimiento del producto potencial o tasa natural.

\section{Método de ajuste de tendencia y elasticidad}

Este método propone estimar para la relación entre producto y desempleo dos aspectos: En primer lugar, que existe una elasticidad constante entre el cociente del PIB observado $(\mathrm{Y})$ y el PIB potencial $\left(\mathrm{Y}^{*}\right)$, respecto del cociente de los niveles de empleo observados (definidos como $\mathrm{N}=100-\mathrm{u}$ ), y los niveles de empleo de pleno empleo $\left(\mathrm{N}^{*}\right)$. Es decir:

$$
\frac{N}{N^{*}}=\left(\frac{Y}{Y^{*}}\right)^{a}
$$

$\mathrm{Y}$, en segundo lugar, asume un crecimiento constante del producto (Y), a partir de un valor inicial (Yo), es decir:

$$
Y_{t}^{*}=Y_{o} e^{r t}(2)
$$

Reemplazando (2) en (1) tenemos:

$$
N_{t}=\frac{\left(Y^{*}\right)^{a} N^{*}}{Y_{o}^{a} e^{a r t}}(3)
$$

Retoma el logaritmo natural de la tasa de empleo con la tendencia temporal y el logaritmo del producto real o efectivo.

$$
\log N_{t}=\log \left(\frac{N^{*}}{Y_{o}^{a}}\right)+a \log Y_{t}-(a r) t
$$

El parámetro a es la elasticidad del empleo ante una variación del producto, y la tasa de crecimiento tendencial del PIB se mide a través del parámetro $r$. 


\section{Material y métodos}

Se parte de la ecuación planteada por Okun, primeras diferencias, para estimar la relación empírica entre producción y empleo utilizando datos desde la década de los 80 hasta el 2014 con frecuencia anual publicados por el Banco Central de Nicaragua, pero teniendo en cuenta que hubo cambios en el cálculo del año base de los datos del PIB real.

Los cálculos efectuados en el desarrollo del modelo se hicieron utilizando el software libre econométrico Gretl disponible en internet.

El modelo es el siguiente:

Tasa de desempleo $=\mathrm{F}($ tasa de variación del PIB, e)

Signo esperado:

El signo esperado de la tasa de variación del PIB es (-)

La ecuación es:

$$
Y=\beta_{0}-\beta_{1} X+e
$$

Donde:

$\mathrm{Y}=$ Tasa de desempleo

$\mathrm{X}=$ Tasa de variación o crecimiento del PIB real.

$\beta_{0}=$ El intercepto

$\beta_{1}=$ Parámetro que mide la magnitud del cambio en $(\mathrm{Y})$.

$\mathrm{e}=\mathrm{el}$ error.

El enfoque estadístico se centra en la introducción de una tendencia estocástica en el modelo de regresión, buscando tomar en cuenta los cambios estructurales de los últimos años en la economía nicaragüense. 
CIENCIAS SOCIALES

\section{Resultados y discusión}

Al correr la regresión mediante mínimos cuadrados ordinarios (MCO), se obtiene el siguiente resultado:

Cuadro 1: Modelo 1: MCO, usando las observaciones 1980-2014 ( $T=35)$

Variable dependiente: Tasa_Desem

\begin{tabular}{|ccccc|}
\hline & Coeficiente & Desv. Típica & Estadístico & Valor $\mathbf{p}$ \\
Const & 7.79607 & 0.833236 & 9.3564 & $<0.00001$
\end{tabular} ***

\begin{tabular}{|llll|}
\hline Media de la vble. dep. & 8.197143 & D.T. de la vble. dep. & 4.230873 \\
Suma de cuad. Residuos & 592.6621 & D.T. de la regresión & 4.237860 \\
R-cuadrado & 0.026203 & R-cuadrado corregido & -0.003306 \\
F(1, 33) & 0.887979 & Valor p (de F) & 0.352873 \\
Log-verosimilitud & -99.17518 & Criterio de Akaike & 202.3504 \\
Criterio de Schwarz & 205.4611 & Crit. de Hannan-Quinn & 203.4242 \\
Rho & 0.894037 & Durbin-Watson & 0.203481 \\
\hline
\end{tabular}

Fuente: Resultados en base a datos del Banco Central de Nicaragua. Utilizando el programa Gretl.

Como se observa los signos no son los esperados. Según el modelo se determina que la propensión marginal a consumir (PMC) es de cerca de o.18, lo que nos indica que a medida que el producto aumenta en un $1 \%$, el desempleo en promedio aumenta también $0.18 \%$. Es decir, como el $\beta$ resultante es positivo en la regresión, las variables se mueven en la misma dirección mostrando una correlación positiva, lo que nos indica que la serie finaliza por debajo de su valor inicial y la otra por encima.

Este resultado pudiera sugerir la presencia de inercia o de histéresis en la tasa de desempleo lo que sería el efecto permanente (o temporal) que tuvo la economía, por los choques negativos transitorios al producto. El efecto histéresis supone que la dinámica del desempleo se explica a partir de valores pasados, lo que contradice la teoría económica tradicional.

Pero que significa ¿histéresis? Este término proviene de la física y el magnetismo, en donde se usa para describir la propiedad que tiene un material o un sistema en los que su estado no depende sólo de las condiciones externas en el instante considerado, sino además de la evolución seguida hasta alcanzar dicho estado. En economía, este término se aplica principalmente en el campo del mercado laboral con el fin de describir la dependencia que tiene la llamada tasa natural de desempleo con su trayectoria en el tiempo. 
la transición de un equilibrio a otro tiende a tener efectos prolongados en la fuerza de trabajo y estos efectos podrían afectar el desempleo de equilibrio por largo tiempo. La tasa natural de desempleo del futuro dependerá del curso de la historia en el interín. Esta propiedad se llama a veces hysteresis (Phelps, 1972, p. 11).

Las economías pueden presentar fenómenos de crecimientos económicos no acompañada por un aumento del empleo. Además, como el coeficiente del producto en el modelo (o.18) es bajo, Blanchard (1997), menciona que esto representa la dificultad que tiene las empresas para adaptarse a los posibles shocks que afectan al producto de la economía.

Cuando el mercado laboral se encuentra con restricciones legales, la estructura interna de las compañías del país, las decisiones políticas, el efecto de la guerra civil que vivió el país en la década de los 70 y 80, donde persisten los efectos de la destrucción del aparato productivo, la gran informalidad del mercado laboral, inciden en la manera en la que el empleo se ajusta ante estos posibles shocks hacia el producto.

Ahora, un punto porcentual de crecimiento en la tasa de desempleo está asociado con una pérdida de $5.53 \%$ en el producto, de acuerdo al inverso del coeficiente $\beta_{1}$ estimado, es decir, $1 / \beta 1$. El coeficiente de correlación $r$ mide sólo la relación lineal entre variables, es decir, es una medida del poder explicativo del modelo lineal; pero no mide la magnitud de la pendiente de la regresión ni si es adecuado un modelo lineal. Si la relación sigue un modelo no lineal (curvado) el coeficiente de correlación puede ser o.

En otras palabras, un coeficiente de correlación pequeño no indica necesariamente que hay poca relación entre las variables, porque puede estar reducido si la relación entre estas variables no es lineal. Una medida de precaución es calcular el intervalo de confianza del coeficiente de correlación $r$ para tener una visión más general, especialmente cuando el número de observaciones sea pequeño.

Cuando los residuos sucesivos están correlacionados positivamente, el valor de D (Durbin Watson $=0.203$ ) se aproximará a cero. Si los resultados no están correlacionados, el valor de D estará cercano a 2. Si se presentase una autocorrelación negativa, lo cual rara vez sucede, el valor $\mathrm{D}$ tomará un valor mayor a 2 e incluso podría aproximarse a su valor máximo que es 4 .

\section{Conclusiones}

Entre 1980-2014 que fue el período analizado, las variables producción y desempleo muestran una relación positiva, es decir, un aumento en la producción de un 1\% trae como consecuencia un aumento en el desempleo de un $0.18 \%$, contradiciendo la teoría económica tradicional donde explica que, si la producción aumenta, necesariamente el 


\section{CIENCIAS SOCIALES}

desempleo disminuye, porque hay más empleo, la economía crece y hay más demanda de mano de obra.

Este fenómeno se presenta porque persiste el efecto de los choques negativos que enfrentó la economía nicaragüense, como la guerra civil que vivió el país en la década de los 80 's y la gran informalidad en el mercado laboral.

\section{Recomendaciones}

Sería interesante evaluar si las series son estacionarias o no estacionarias, es decir si presentan raíces unitarias. Si se incluyeran series que no son estacionarias puede conducir a resultados falsos en los Modelos de Mínimos Cuadrados Ordinarios (MCO). Se puede aplicar test de Dickey-Fuller Aumentada y de Phillips Person, para comprobar si existe una relación tendencial entre las variables.

La posibilidad de que el empleo o desempleo en el período analizado se encuentre explicado por factores diferentes al Producto Interno Bruto (PIB), son alternativas que están fuera del alcance de este modelo. Sin embargo, esta posibilidad merece atención para este caso, donde se puede ampliar el modelo, siguiendo las otras alternativas que presenta Okun. Tampoco se debe obviar el mecanismo de corrección de errores que sirve para reconciliar el comportamiento de corto plazo de una variable económica con su comportamiento de largo plazo.

\section{Lista de referencias}

Abril, J., Ferullo, H., y Gainza, A. (1996). Estimación de la relación Okun: Argentina 1980-1996. Universidad de Tucumán.

Arango, L. y Posada, E. (2003). El desempleo en Colombia. Borradores de Economía. Banco de la República. Disponible en : http://www.banrep.org/docum/investi4.htm.

Blanchard, O. (1997). Macroeconomía. Prentice Hall, Madrid.

Blanchard O, y Pérez D. (1980). Macroeconomía: Teoría y Política Económica con aplicaciones a América Latina. Disponible en: http:/librería-universitaria.blogspot.com

Banco Central de Nicaragua (2011). Nicaragua en Cifras. Disponible en http:/www. bcn.gob.ni.

Banco Central de Nicaragua (2013),.Información Estadística. Disponible en http:/www. bcn.gob.ni.

Damodar, G. y Dawn, P. (2009). Econometría (5ta. ed). México. The McGraw-Hill. 
Franklin O. (2016). Aproximación a la Relación PIB Empleo Desempleo (tesis doctoral). Universidad del Zulia, Venezuela.

Mochón M. (2006). Principios de Macroeconomía. España: McGraw-Hill. 2da. Ed.

Martin, M. y Doris, N, (1994). La histéresis en el desempleo colombiano. Cuaderno de Economía, v XIV, n. 21, Bogotá.

Liquitaya J. y Lizarazu E. (1985). La ley de Okun en la Economía Mexicana. Revista denarius.

Latorre, M. (1983). Respuesta del Desempleo a Variaciones del Producto: Cuantificaciones para Ecuador a partir de la ley de Okun. Documento de trabajo.

Okun, A. (1962). Potential GNP: its measurement and significance (Potencial PNB: su medida y significado). Reprinted From the 1962 proceeding of the business and Economic Statistic Section of the American Statistical Association, Cowles Foundation paper 190.

Orsini, G. y Rusteholz, G. (2009). El fenómeno de la histéresis del desempleo en el mercado laboral Argentino". Presentada en la XLIV Reunión Anual, Asociación Argentina de Economía Política. Disponible en http://www. aaep.org.ar

PHELPS, E (1972). The evolution of unemployment in the United States: 1968-1985 (La evolución del desempleo en los Estados Unidos: 1968-1985). En: NBER Macroeconomics Anual. Vol 2, 1972, p. 11-58.

Tobin, J. (1980). Asset Accumulations and Economic Activity, Basil Blackwell, Oxford (Acumulación de activos y actividad económica, Basil Blackwell, Oxford).

Tobin, J. (1980). In Memoriam. A tribute to Arthur M. Okun (En memoria. Un tributo a Arthur M. Okun). The Brookings Institution Washington. 